\title{
5. DOCUMENTATION AND ASTRONOMICAL DATA (DOCUMENTATION ET DONNEES ASTRONOMIQUES)
}

PRESIDENT: B. Hauck

VICE PRESIDENT: O. Dluzhnevskaya

ORGANIZING COMMITTEE: M. Crézé, P. Dubois, J. Grosbol, A. Heck, Li Qi-Bin J. Mead, L. Schmadel, K. Turner, W.H. Warren, G. Westerhout, G. Wilkins

\section{INTRODUCTION}

The scope of our commission may appear very broad, with ill-assorted topics. However, this is a wrong impression and $I$ think that in the very near future the convergence of all our activities will appear clearer. With the development of computer resources and possibilities, all our activities lead to what we could call information science. Two books published lately (Intelligent Infomation Retrieval, A. Heck \& F. Murtagh, Eds., Kluwer Acad. Publ., 1993 and Databases and On-line Data in Astronomy, M. Albrecht \& D. Egret, Eds., Kluwer Acad. Publ., 1991) give a very good idea of this tendency.

Dr. A. Heck was the IAU representative to the General Assembly of CODATA, held in Beijing in October 1992. The next General Assembly will take place in Chambéry, France, 18-22 September 1994. The scientific theme is Data and Knowledge in a Changing World.

The past triennium has been marked by a lot of activities related to Commission 5, the major ones being listed below. I would just emphasise at this point those activities which I consider to be most important.

First is the archiving of current observational data. A JCM was foreseen during the last General Assembly in Buenos Aires, but due to the fire which broke out at the San Martin Cultural Center, it was not possible to hold it. However, the proceedings are published in Highlights of Astronomy, 9, 1992. In some observatories [53.013.039, 53.013.040, 53.013.041] steps have been taken to organise observational archives. The proceedings of a workshop on this topic held in April 1993 at Trieste will be published soon by M.A. Albrecht and F. Pasian.

Next may be mentioned as an important change the apparition of two information systems ESIS [53.013.042] and ADS [53.013.043], the latter being more developed at the time of the preparation of this report than the first.

The last point I would mention is the astronomical literature that is accessible on-line. The main project in this field is STELLAR (Study of Electronic Literature for Astronomical Research), a project developed at GSFC which provides abstracts of articles in ApJ, ApJS, AJ, PASP, A\&A, AAS, MN and JGR.

\section{WORKSHOPS, COLLOQUIUMS, CONFERENCES}

Various meetings relating to the scope of Commission 5 were held, of which the following may be mentioned: 
Desktop Publishing in Astronomy and Space Sciences, Strasbourg, 1991 [55.012.053]

Astronomy from Large Databases II, Haguenau, 1992 [A. Heck \& F. Murtagh (Eds.)]

Handling and Archiving Data from Ground-based Telescopes, Trieste, 1993

Astronomical Data Analysis Software and System I, Tucson, 1991 [D.M. Worrall et al. (Eds.)]

Astronomical Data Analysis Software and System II, Boston, 1992 [R.J. Hanisch et al. (Eds.)]

Astronomical Data Analysis Software and System III, Victoria, 1993

Databases for Galactic Structure, Swarthmore, 1993 [A.G.D. Philip et al. (Eds.)]

\section{REPORTS FROM DATA CENTRES}

\subsection{NASA ADC (by N. Roman)}

The NASA ADC produced and distributed more than 2000 copies of the first ADC CDROM which contained 114 catalogues in ASCII and FITS formats. The ADC prepared new printed versions of the Bonner Durchmusterung, the Cordoba Durchmusterung, the Cape Photographic Durchmusterung and the WDS/HD/DM/ADS crossindex.

As of April, 1993, the NASA ADC had 669 catalogues in its archive of which 82 were added since July 1991 , besides those received from other data centres. The ADC is cooperating with the CDS in archiving smaller data sets by acquiring these from North American publications. In addition to the ADC CDROM, the NSSDC is distributing CDROMs of data from the Einstein Observatory, the IRAS Sky Survey Atlas, and Halley and planetary observations.

\subsection{CDS, Strasbourg (by M. Crézé)}

Over the last two years the CDS activity has been developing along its usual lines of action, namely:

\section{The Simbad Database}

Now widely used as a reference for cross-identification of astronomical objects, it provides cross-identifications, bibliographic references and a selection of basic data for over 1 million astronomical objects including galactic and extra galactic sources observed at various wavelengths.

- Improvements to the new software are going on, including assistance to Simbad users and updates of the user's guide.

- The bibliographical effort has been continued, references to astronomical objects are available nearly at publication time.

- There are 880 registered accounts actually used. The number of countries with Simbad users was 24 by the fall of 1990,36 by the fall of 1992 . 
- Provisional free access has been open for countries with currency difficulties.

- A major update of the Simbad content has been the cross-identification of the IRAS Point Source Catalogue.

- New developments currently investigated : graphical user interface, Computer Assisted Quality Control.

\section{The catalogue exchange and distribution facility}

- Over 80 new catalogues have been incorporated in the CDS files.

- A new facility has been implemented to make most catalogues (and all the recent ones) freely available through an anonymous ftp procedure, being the currently most widely used process.

- From January 1993 onwards, an agreement has been passed with the editor of the journal "Astronomy and Astrophysics" to incorporate in the CDS files data related to papers published in "Astronomy \& Astrophysics Supplements" and to make them available via the ftp procedure.

- Experience has been gained in re-digitising printed data through scanner and character recognition, which will greatly help to create new compilations.

\section{Methodologies and techniques for information retrieval}

The CDS has published "Star Guides" : A Directory of Astronomy, Space Sciences and Related Organisations of the World (1993, CDS Special Pubs $n^{\circ} 20$ ), and "Star Briefs" Dictionary of Abbreviations, Acronyms and Symbols in use in Astronomy, Space Sciences and related fields (1993, CDS Special Pubs $\mathrm{n}^{\circ} 21$ ).

\section{International Co-operation}

The CDS has been permanently fostering the exchange of experience and data between the 7 Astronomical Data Centres inside the working group created by the IAU. Main efforts have been dedicated to bring computer-readable data availability up to equivalent levels in all parts of the world. This is far from being achieved mainly due to the uneven level of technical development. Current efforts aim at creating highly compatible tools for handling, analysing and formatting new data sets in a number of ADCs. Such tools would enable Data Centres to share the task of incorporating and distributing data sets into and from their own archives and eventually create mirror archives at different places.

The CDS also collaborates in the development of new facilities to access data such as the ESIS project of ESA and the ADS project of NASA. 


\subsection{Astronomical Data Analysis Center, National Astronomical Observatory of Japan (by S. Nishimura)}

A data storage and distribution system for astronomical catalogues was developed on UNIX workstations based on magneto-optical disks. The older system on openreel magnetic tapes is also running on the main-frame computer.

Validation, documentation and reorganisation of astronomical catalogues, especially those made in Japan, have been done at the Center. A CD-R writer was recently installed at the Center, and it is planned to distribute custom-made sets of catalogues, such as the Photometric Atlas of Galaxies or data sets of special fields of astronomy.

\subsection{Centre of Astronomical Data, Institute of Astronomy of the Russian Academy of Sciences (INASAN) (by O. Dluzhnevskaya)}

The Centre (CAD) was established as a branch centre of the Strasbourg Data Centre (CDS) and has been active since 1977 on the basis of the Agreement signed by both Centres.

The CAD is responsible for data activity and coordination in the former SU and provides the CDS with new catalogues and other astronomical data prepared at different astronomical institutes.

Since 1980 the World Data Center A of the Goddard Space Flight Center (ADC) and $\mathrm{CAD}$ have also begun to exchange machine-readable versions of astronomical catalogues.

In the frame of the agreements, the CAD is responsible for the preparation of machine-readable astronomical catalogues, software etc. in the FSU.

During the last 3 years 11 astronomical catalogues were sent to the CDS and 9 are ready.

The CAD participated in the preparation of the list of astrophysical catalogues on CD-ROM prepared by the ADC and after receiving the CD-ROMs (Test disk, Selected Astronomical Catalogs, vol. 1) they were disseminated throughout the astronomical observatories and institutes of the former SU.

The CAD provides the CDS and ADC with information about the preparation of new catalogues compiled in observatories of the FSU.

During the past year a compilation was made of different astronomical data sets in the form of archives, data bases (already existing and those being created), miscellaneous astronomical data sets and plate vaults stored at the various observatories. This compilation contains information about the instruments used for observations, areas of the sky and time intervals they cover, photometric systems, limiting stellar magnitudes and computer facilities available at the observatories. It has been sent to the CDS for publication.

The Center has a present staff of 12 people, using 4 work stations and 5 PCs connected via the LAN Ethernet.

Projects in progress include: completion of a machine-readable version of the catalogue of observational data of star-forming regions; the creation of an updated collection of observational data of stars in stellar associations; studies in the field of galactic stellar population evolution (initial mass function, star clusters, young stellar complexes); 26 papers on this subject were published this year. 


\subsection{Centro de Datos Astronomicos, Argentina (by E.F. Arias)}

The Centro de Datos Astronomicos Argentina (CDA) is installed at the Facultad de Ciencias Astronomicas y Geofisicas de La Plata, Argentina. It works under the responsibilitiy of two local astronomers, Dr. Elisa Felicitas Arias and Lic. Horacio Luis Tignanelli. It was created by means of an agreement between the Universidad Nacional de La Plata and the Strasbourg Stellar Data Centre. The CDA distributes astronomical data to all Argentine observatories. It also provides on request information to some South American colleagues. Periodically the CDA sends by e-mail information bulletins to Argentine users.

\section{REPORTS FROM WORKING GROUPS AND TASK GROUPS}

Before reporting on the activity of the Commission's WGs and TGs I would mention the activity of two WGs of two other commissions that receive our support. The first is the WG on "Radioastronomical Databases" (Commission 40), chaired by Dr. H. Andernach, IAC. This WG held its first WG meeting (ESO-OAT Workshop in Trieste in April 1993). Recommendations emerged on electronic availability of published data, on the educational needs for the age of "Network Astronomy", the preparation of a master index of all machine-readable astronomical catalogues, and the nomenclature of radio sources. Electronic access to radio source catalogues has been vastly improved by the WG: 45 tables $(\sim 420,000$ entries) are now searchable on-line in the "Einstein On-line Service" and 40 more tables ( 80,000 entries) are in the waiting list. With Commissions 5 and 29 it is proposed to hold an "Interdisciplinary Discussion on the Status of Archiving of Astronomical Data" at the GA in 1994.

The second is the WG on Spectroscopic Data Archives, chaired by Dr. E. Griffin, which was established in mid-1992, its raison d'être having been enshrined in IAU Resolution C13 (1991). The 12 members come from all continents except Antarctica; most are research astronomers who are personally committed to the benefits of archiving, and who try to encourage, stimulate, rationalize and sometimes help organise suitable archiving policies in all observatories and institutions. The WG took an active part in various workshop meetings both in Europe and the USA and was especially in evidence at the workshop in Trieste (April 1993), where its own EC grant helped support the attendance of 19 people. The WG is currently tackling the cataloguing of spectroscopic plates and is considering the idea of putting all such plates in one place as a long-term safeguard.

The Astronomy Thesaurus Task Group, headed by Mrs. Robyn Shobbrook, has published an astronomical thesaurus. Its main aim is to standardize the terminology for the purposes of aiding unambiguous library cataloguing and more precise recall of data from computer databases. The thesaurus can be ordered directly from Mrs. R. Shobbrook, Anglo-Australian Observatory, Epping Laboratory, P.O. Box 296, 296 Epping N.S.W. 2121, Australia.

The WG on Libraries, co-chaired by Dr. W.H. Warren and Mrs. B. Corbin, is now planning a conference on Library and Information Services in Astronomy (LISA II) 
The WG on Designations, chaired by Dr. P. Dubois, has been very active since the last $\mathrm{GA}$, in two directions.

First, a new version of the "Dictionary of Nomenclature of Astronomical Objects", compiled by M.-C. Lortet and collaborators, is a very important tool in the field of designation. It is now possible to access it on-line on the SIMBAD computer (simbad.u-strasbg.fr or 130.79 .128 .4 ) at Strasbourg and without password under the login "info". Also to be found there is other information such as the IAU document on "Specifications...". The dictionary is also on-line in the SIMBAD database and is regularly updated. A printed version will also be available at the end of the year. Designation problems were also the subject of discussion during the Trieste meeting.

Second, the WG has been active in promoting the IAU Specifications (see e.g. A\&A 267, A12, 1993). This document should serve as a reference for problems encountered in the designation of astronomical objects. A new version is now in preparation and the chairman of the WG welcomes all suggestions. 